\title{
Introduction to Digital Location Minitrack
}

\author{
Jim Thatcher \\ University of Washington - Tacoma \\ jethatch@uw.edu
}

\author{
Britta Ricker \\ University of Washington - Tacoma \\ bricker0@uw.edu
}

The purpose of this minitrack is to comprehensively engage with research that investigates digital locational data. We call for papers that address the production, capture, and study of location information through both technical and theoretical perspectives. Research into the processes associated with data capture and analysis, including visualization techniques, is in high demand. This is in part due to the ever- growing production of locational data via emerging spatially-aware technologies, such as smartphones, personal fitness trackers, and unmanned aerial vehicles (UAVs).

Here we invite papers that address 'location information' in a broad sense that includes both precise geolocated coordinates and more general expressions of space and place. Potential data sources include individuals and industries - for example, government open data initiatives, personal activity trackers, social media services, or aerial photography from UAVs. We wish to engage with scholarship that addresses the technical considerations around working with locational data as well as its transformation into locational media.

This includes, but is not limited to, papers that: offer new technical and methodological solutions to the capture, interpretation, analysis or visualization of spatial media; examine the epistemological and ontological effects of spatial social media upon users; present empirical work on the creation or consumption of spatial social media; advance our understanding of how spatial social media relate to social and political processes; present new work on the role of economic forces in the creation and use of spatial social media, for example, location-specific advertising; or explore spatial social media as a means of better understanding urban and non-urban environments. More specifically, we encourage papers that engage with the following topics or related areas:
- $\quad$ Spatial Informatics, data mining and data exploration of spatial information

- Crowdsourced spatial information

- New or emerging locational data collection techniques

- Resistance and/or surveillance through spatial digital information and social media

- Mapping social media for humanitarian efforts

- FOSS technologies for location aware research

- Social media and citizen science initiatives

- Governmental Open Data analyzed and distributed through social media

- Gendered representations in spatial digital information and social media

- Ethical considerations associated with the use of spatial digital media for information sharing

- Scale and information relevance related to social media networks and location

- Qualitative research on the use of spatial social media by end-users and firms

- New or alternative methodological techniques for the collection, analysis and visualization of spatial social media information 